\title{
BEARING CAPACITY ANALYSIS USING MESHLESS LOCAL PETROV-GALERKIN METHOD
}

\author{
Juraj MUŽík ${ }^{1, *}$ \\ ${ }^{1}$ Department of Geotechnics, Faculty of Civil Engineering, University of Žilina, Žilina, Slovak Republic. \\ * corresponding author: juraj.muzik@fstav.uniza.sk.
}

\begin{abstract}
The paper deals with use of the meshless method for soil bearing capacity analysis. There are many formulations of the meshless methods. The article presents the Meshless Local Petrov-Galerkin method (MLPG) - local weak formulation of the equilibrium equations. The main difference between meshless methods and the conventional finite element method (FEM) is that meshless shape functions are constructed using randomly scattered set of points without any relation between points. The Heaviside step function is test function used in the meshless implementation presented in the article. Heaviside test function makes weak formulation integral very simple, because only body integral in governing equation is due a body force.
\end{abstract}

\section{Keywords:}

Bearing capacity; Heaviside step function; Meshless local Petrov-Galerkin method;

Meshless methods;

Numerical methods.

\section{Introduction}

In the Local Petrov-Galerkin method, the problem domain is represented by a set of arbitrarily distributed nodes. The weighted residual method is used to create the discrete system equation. The weighted residual method is, of course, in integral form, and a background mesh of cells is still required for the integration. The major idea in MLPG is, however, that the implementation of the integral form of the weighted residual method is confined to a very small local subdomain of a node. This means that the weak form is satisfied at each node in the problem domain in a local integral sense. Therefore, the weak form is integrated over a "local quadrature domain" that is independent of other domains of other nodes. This is made possible by use of the Petrov-Galerkin formulation, in which the weight and trial functions can be chosen independently.

\section{Shape functions in meshless sense - Moving least squares method (MLS)}

The creation of the shape or trial functions is the crucial part of meshless numerical analysis. The moving least squares method (MLS) is used to construct the shape function based on set of scattered nodes. There is no predefined relationship between nodes such as an element in finite element analysis.

Consider the task of finding an approximate solution $u^{h}(x)$, knowing the true solution, $u_{a}$ at selected points $x_{a}$. Then in a least squares sense minimization of the expression $\left[u^{h}\left(x_{a}\right)-u_{a}\right]^{2}$ for each $a$ is the objective. Suppose a polynomial approximation is chosen so that

$$
u^{h}(x)=g_{1}+g_{2} x+g_{3} x^{2}+\ldots g_{m+1} x^{m}
$$


The approximation is then written in matrix form

$$
u^{h}(x)=\mathbf{p}^{\mathbf{T}}(x) \mathbf{g}=\left[\begin{array}{lllll}
1 & x & x^{2} & \ldots & x^{m}
\end{array}\left[\begin{array}{c}
g_{1} \\
g_{2} \\
g_{3} \\
\vdots \\
g_{m+1} x^{m}
\end{array}\right] .\right.
$$

Using the above the least squares functional is written with the approximation substituted in for $u^{h}(x)$

$$
J=\frac{1}{2} \sum_{a=1}^{n}\left\{u^{h}\left(x_{a}\right)-u_{a}\right\}^{2}=\frac{1}{2} \sum_{a=1}^{n}\left\{\mathbf{p}^{\mathbf{T}}\left(x_{a}\right) \mathbf{g}-u_{a}\right\}^{2}
$$

Now, recall that compact support for each node $a$ is intended. Therefore, the local solution is influenced by the local nodes. Whereas, nodes far away have no influence. Hence, each summation term, indexed by $a$, in the least squares functional is weighted by a weight function $w_{a}$, which limits the term's influence to point $a$ and usually several surrounding nodes. Based on this intuition the functional $J$ is modified and becomes a weighted least squares functional as follows

$$
J=\frac{1}{2} \sum_{a=1}^{n} w_{a}\left\{\mathbf{p}^{\mathbf{T}}\left(x_{a}\right) \mathbf{g}-u_{a}\right\}^{2}
$$

Next, it is necessary to minimize $J$ with respect to each $g_{i}$. However, before this operation, it is helpful to write the functional in matrix form. Then the functional $J$ is written as:

$$
J=\frac{1}{2}(\mathbf{P g}-\mathbf{u})^{T} \mathbf{W}(\mathbf{P g}-\mathbf{u})
$$

where

$$
\mathbf{P}=\left[\begin{array}{cccc}
p_{1}\left(x_{1}\right) & p_{2}\left(x_{1}\right) & \ldots & p_{k}\left(x_{1}\right) \\
\vdots & \ddots & & \vdots \\
\vdots & & \ddots & \vdots \\
p_{1}\left(x_{n}\right) & p_{2}\left(x_{n}\right) & \ldots & p_{k}\left(x_{n}\right)
\end{array}\right]
$$

Notice that each row of the $\mathbf{P}$ matrix is just $p^{T}\left(x_{a}\right)$ for each row $a$. And, the size of this matrix is $n$ by $k$. 
For $\mathbf{W}$, an $n$ by $n$ matrix results

$$
\mathbf{W}=\left[\begin{array}{cccc}
w_{1}\left(x-x_{1}\right) & & & 0 \\
& w_{2}\left(x-x_{2}\right) & \\
& & \ddots & \\
0 & & & w_{1}\left(x-x_{n}\right)
\end{array}\right],
$$

where the diagonal terms $w_{a}$ are defined as $4^{\text {th }}$ order quartic spline function with $q=\left|x-x_{a}\right| / \rho_{a}$

$$
w(q)=\left\{\begin{array}{cc}
1-6 q^{2}+8 q^{3}-3 q^{4} & q \leq 1 \\
0 & q \succ 1 .
\end{array}\right.
$$

The $\mathbf{g}$ vector is $k$ by 1 and the $\mathbf{u}$ vector is $n$ by 1 .Now, set $\partial \mathrm{J} / \partial \mathrm{g}=0$. This yields the following

$$
(\mathbf{P g}-\mathbf{u})^{T} \mathbf{W P}=0
$$

Transposing the whole equation yields

$$
(\mathbf{W P})^{T}(\mathbf{P g}-\mathbf{u})=0
$$

Multiplying through gives

$$
\mathbf{P}^{\mathrm{T}} \mathbf{W P g}-\mathbf{P}^{\mathrm{T}} \mathbf{W u}=0
$$

and finally

$$
\mathbf{P}^{\mathrm{T}} \mathbf{W P g}=\mathbf{P}^{\mathrm{T}} \mathbf{W u}
$$

Now define the moment matrix $\mathbf{A}=\mathbf{P}^{\top} \mathbf{W} \mathbf{P}$ and $\mathbf{B}=\mathbf{P}^{\top} \mathbf{W}$. Note that $\mathbf{A}$ is $k$ by $k$ and $\mathbf{B}$ is $k$ by $n$. Using these definitions Eq. (12) becomes

$$
\mathbf{A g}=\mathbf{B u}
$$


Solve now for the unknown coefficients $\mathbf{g}$

$\mathbf{g}=\mathbf{A}^{-1} \mathbf{B u}$

Substitute this into the first part of Eq. (...)

$u^{h}=\mathbf{p}^{T}(x) \mathbf{g}=\mathbf{p}^{T}(x) \mathbf{A}^{-1} \mathbf{B u}$.

The approximations $u^{h}$ are usually written as

$u^{h}=\boldsymbol{\varphi}^{T} \mathbf{u}=\sum_{a=1}^{n} \phi_{a} u_{a}$

Comparison of Eq. (16) with Eq. (15) reveals that the vector of MLS shape functions is

$\boldsymbol{\varphi}^{T}=\mathbf{p}^{T}(x) \mathbf{A}^{-1} \mathbf{B}$

Notice that the $\mathbf{A}$ and $\mathbf{B}$ matrices depend on $\mathbf{W}$. The $\mathbf{W}$ matrix in turn is a function of the $x_{a}$ and the evaluation point $x$. Hence every time a new evaluation point $x$ is chosen the matrices $\mathbf{A}^{-1}$ and $\mathbf{B}$ are recomputed to calculate the MLS shape functions based on the Equation (17).

\section{Weak formulation of equilibrium equations}

Consider a solid mechanics boundary problem defined over domain $\Omega$. For a field node $I$, the governing equation is satisfied using a locally weighted residual method, leading to a weak form equation for this node. The local weighted residual form defined over a local quadrature domain $\Omega_{q}$ bounded by $\Gamma_{\mathrm{q}}$ is defined as

$\int_{\Omega_{q}} W_{I}\left(\sigma_{i j, j}+b_{i}\right) \mathrm{d} \Omega=0$,

where $W_{l}$ is the test function usually centered at node $I$. Equation (18) is applied to all the nodes in the domain $\Omega$.

When the local weighted residual formulation rather than the global energy principle is used to create discretized equation system node by node, the compatibility of the shape functions in whole domain is not required. As long as the field approximation is continuous at any point in the local quadrature domain $\Omega_{\mathrm{q}}$, the shape function is differentiable and the resultant integrand is integrable, the solution will exists. So the local weak-form method only requires the local compatibility in the local quadrature domain $\Omega_{\mathrm{q}}$. The RPIM shape function satisfies all these requirements and brings advantage of its delta function property. The first term on the left hand side of the Equation (18) can be integrated by parts

$\int_{\Omega_{q}} W_{I} \sigma_{i j, j} \mathrm{~d} \Omega=\int_{\Gamma_{q}} W_{I} n_{j} \sigma_{i j} \mathrm{~d} \Gamma-\int_{\Omega_{q}} W_{I, j} \sigma_{i j} \mathrm{~d} \Omega$, 
where $n_{j}$ is the th component of the unit outward normal on the boundary. Substituting Equation (19) back to the Equation (18) the following form is obtained

$\int_{\Gamma_{q}} W_{I} \sigma_{i j} n_{j} \mathrm{~d} \Gamma-\int_{\Omega_{q}}\left[W_{I, j} \sigma_{i j}-W_{I} b_{i}\right] \mathrm{d} \Omega=0$

The boundary of the local quadrature domain is composed by three parts $\Gamma_{q}=\Gamma_{q i} \cup \Gamma_{q u} \cup \Gamma_{q t}$ (Fig.1) where

- $\Gamma_{\mathrm{qi}}$ is the internal boundary of the quadrature domain, which does not intersect with the global boundary $\Gamma$;

- $\quad \Gamma_{\mathrm{qt}}$ is the part of the natural boundary that intersect with the quadrature domain;

- $\Gamma_{\mathrm{qu}}$ is the part of the essential boundary that intersect with the quadrature domain.

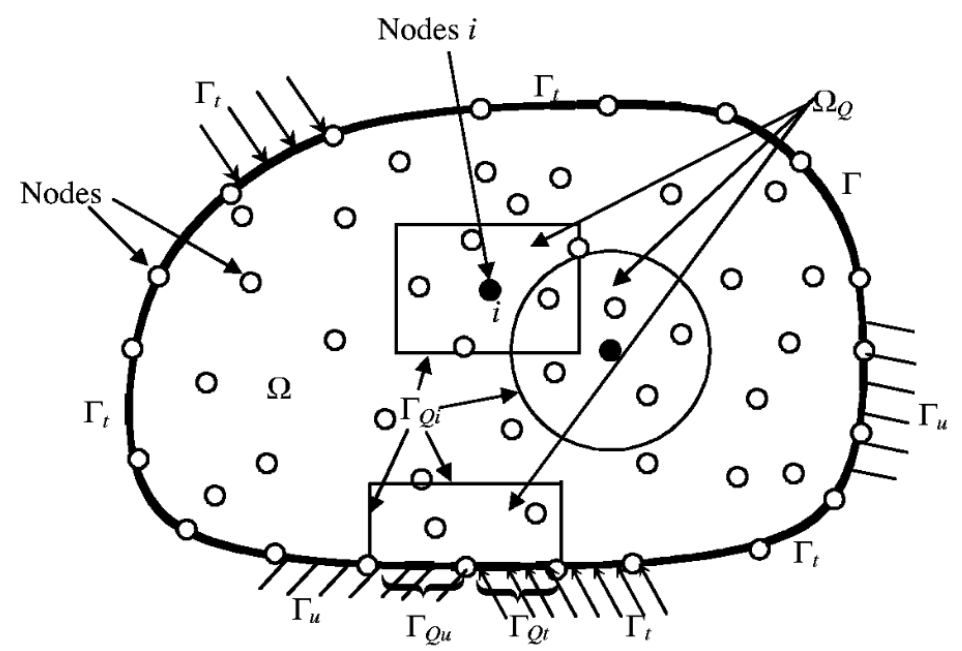

Fig.1: Domains and their boundaries. Global boundary $\Gamma$ including essential (displacement) boundary $\Gamma_{u}$, natural (force or free) boundary $\Gamma_{\mathrm{t}}$; quadrature domain of $\Omega_{\mathrm{Q}}$ and its boundary including the interior boundary $\Gamma_{\mathrm{Qi}}$ that is located within the problem domain, the essential boundary $\Gamma_{\mathrm{Qu}}$ that intersects with $\Gamma_{\mathrm{u}}$ and natural boundary $\Gamma_{\mathrm{Qt}}$ that intersects with $\Gamma_{\mathrm{t}}$.

Therefore, the equation (20) can be rewritten as

$\int_{\Gamma_{q i}} W_{I} \sigma_{i j} n_{j} \mathrm{~d} \Gamma-\int_{\Gamma_{q u}} W_{I} \sigma_{i j} n_{j} \mathrm{~d} \Gamma-\int_{\Gamma_{q t}} W_{I} \sigma_{i j} n_{j} \mathrm{~d} \Gamma-\int_{\Omega_{q}}\left[W_{I, j} \sigma_{i j}-W_{I} b_{i}\right] \mathrm{d} \Omega=0$.

For the local quadrature domain located entirely within global domain, there is no intersection between $\Gamma_{\mathrm{q}}$ and the global boundary $\Gamma$. For such a case there is no integral over $\Gamma_{\mathrm{qt}}$ and $\Gamma_{\mathrm{qu}}$ and Equation (21) is simplified to

$\int_{\Gamma_{q i}} W_{I} \sigma_{i j} n_{j} \mathrm{~d} \Gamma-\int_{\Omega_{q}}\left[W_{I, j} \sigma_{i j}-W_{I} b_{i}\right] \mathrm{d} \Omega=0$.

In this local weak form, (Eqs. (21) and (22)) the Petrov-Galerkin method can be used, in which the trial and test functions are selected from different function spaces. Because the resulting integrals 
are complicated there is need to simplify them by proper choice of the test function. The promising approach is to use the Heaviside step function as the test function. The Heaviside function (23) is defined as value of 1 over the quadrature domain and 0 outside.

$W_{I}=H_{I}[\mathbf{x}]= \begin{cases}0 & \mathbf{x} \notin q \\ 1 & \mathbf{x} \in q\end{cases}$

Using the Heaviside step function as the test function, the volume integrals in the Equations (21) and (22) vanish except those for the body force term, resulting in following formulation

$$
\int_{\Gamma_{q i}} \sigma_{i j} n_{j} \mathrm{~d} \Gamma-\int_{\Gamma_{q u}} \sigma_{i j} n_{j} \mathrm{~d} \Gamma=\int_{\Gamma_{q t}} \sigma_{i j} n_{j} \mathrm{~d} \Gamma-\int_{\Omega_{q}} b_{i} \mathrm{~d} \Omega
$$

and for the quadrature domain completely within the global domain

$$
\int_{\Gamma_{q i}} \sigma_{i j} n_{j} \mathrm{~d} \Gamma=-\int_{\Omega_{q}} b_{i} \mathrm{~d} \Omega
$$

Basically this formulation is similar to the local boundary element formulation (LBIE), except that no fundamental solution and no special care is needed for singularities. To obtain the matrix form of the governing equation in the terms of displacement $\mathbf{u}$ following notation is used

$$
\mathbf{u}(\mathbf{x})=\left[\begin{array}{ccccc}
\phi_{1} & 0 & \cdots & \phi_{n} & 0 \\
0 & \phi_{1} & \cdots & 0 & \phi_{n}
\end{array}\right]\left[\begin{array}{c}
u_{1} \\
v_{1} \\
\vdots \\
u_{n} \\
v_{n}
\end{array}\right],
$$

where $\varphi_{i}$ is nodal shape function used to interpolate a displacement field in the terms of nodal displacements $\left(u_{i}, v_{i}\right)$. Next the relation between the displacement field and strains should be defined in the context of geometrically linear (infinitesimal) deformation theory

$$
\boldsymbol{\varepsilon}=\mathbf{B u}
$$

where strain-displacement matrix $\mathbf{B}$ is defined using shape functions derivatives with respect to spatial coordinates 


$$
\mathbf{B}=\left[\begin{array}{ccccc}
\frac{\partial \phi_{1}}{\partial x} & 0 & \cdots & \frac{\partial \phi_{n}}{\partial x} & 0 \\
0 & \frac{\partial \phi_{1}}{\partial y} & \cdots & 0 & \frac{\partial \phi_{n}}{\partial y} \\
\frac{\partial \phi_{1}}{\partial y} & \frac{\partial \phi_{1}}{\partial x} & \cdots & \frac{\partial \phi_{n}}{\partial y} & \frac{\partial \phi_{n}}{\partial x} \\
0 & 0 & \cdots & 0 & 0
\end{array}\right] .
$$

The stress components are obtained from the constitutional equation defined using standard elastic matrix D

$\boldsymbol{\sigma}=\mathbf{D} \boldsymbol{\varepsilon}$

where matrix $\mathbf{D}$ is defined in terms of Young modulus $E$ and Poisson number v

$\mathbf{D}=\frac{E(1-v)}{(1+v)(1-2 v)}\left[\begin{array}{cccc}1 & \frac{v}{1-v} & 0 & \frac{v}{1-v} \\ \frac{v}{1-v} & 1 & 0 & \frac{v}{1-v} \\ 0 & 0 & \frac{1-2 v}{2(1-v)} & 0 \\ \frac{v}{1-v} & \frac{v}{1-v} & 0 & 1\end{array}\right]$.

Finally the boundary traction $\mathbf{t}$ is defined using outward unit vector components and stress components

$\mathbf{t}=\mathbf{n} \boldsymbol{\sigma}=\left[\begin{array}{cccc}n_{x} & 0 & n_{y} & 0 \\ 0 & n_{y} & n_{x} & 0\end{array}\right]\left[\begin{array}{c}\sigma_{x} \\ \sigma_{y} \\ \tau_{x y} \\ \sigma_{z}\end{array}\right]$.

Substituting (28), (30) and (31) into (24) and (25) the following formulations are obtained

$$
\begin{aligned}
& \int_{\Gamma_{q i}} \mathbf{n D B u d} \Gamma-\int_{\Gamma_{q u}} \mathbf{n D B u d} \Gamma=\int_{\Gamma_{q t}} \mathbf{t d} \Gamma-\int_{\Omega_{q}} \mathbf{b d} \Omega, \\
& \int_{\Gamma_{q i}} \mathbf{n D B u d} \Gamma=-\int_{\Omega_{q}} \mathbf{b d} \Omega .
\end{aligned}
$$




\section{Numerical example of the bearing capacity calculation}

The bearing capacity analysis performed by MLPG use the elastic-perfectly plastic stress-strain law with Tresca failure criterion (34).

$F=\frac{\bar{\sigma} \cos \theta}{\sqrt{3}}-c_{u}$,

where $\sigma$ and $\theta$ are the second and third stress invariant and $c_{u}$ is the undrained cohesion of the soil. The plastic stress redistribution is accomplished using "initial stress" algorithm. The MLPG implementation uses regular node field generated with nodal spacing $d_{c}=0.5[\mathrm{~m}]$. The model consists of 340 nodes regularly distributed over rectangular domain defined by width $w_{x}=50[\mathrm{~m}]$ and height $w_{y}=17[\mathrm{~m}]$ and represents the undrained clay layer. In the middle of the model upper boundary the strip footing with length of $b=6[\mathrm{~m}]$ is located. The loading stress $q$ is increased incrementally to failure. The elasto-plastic soil is described by three parameters, namely the Young modulus $E$, Poisson number $v$ and the undrained cohesion $c_{u}$. Bearing failure in this domain occurs when $q$ reaches Prandtl load given by

$q_{U L T}=(2+\pi) c_{u}$.

The rectangular support domain used for interpolation has half-size $d_{s}=2.9 d_{c}=1.45[\mathrm{~m}]$. The quadrature domain used for numerical integration is composed as very simple mesh of the rectangular shape with half size $r_{q}=2.1 d_{c}=1.05[\mathrm{~m}]$. The quadrature domain is divided into 4 regular sections with $16(4 \times 4)$ Gauss points in each section.
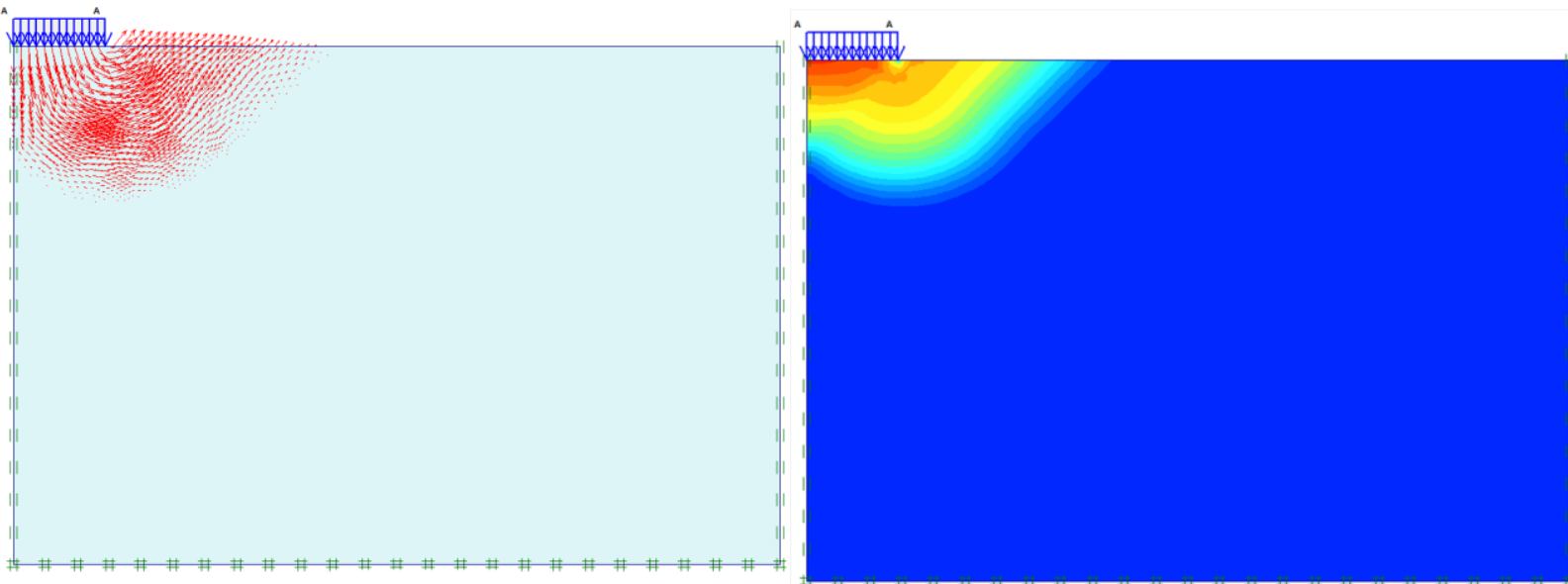

Fig. 2: Displacements as vectors and shadings at failure computed by FEM (Plaxis). 

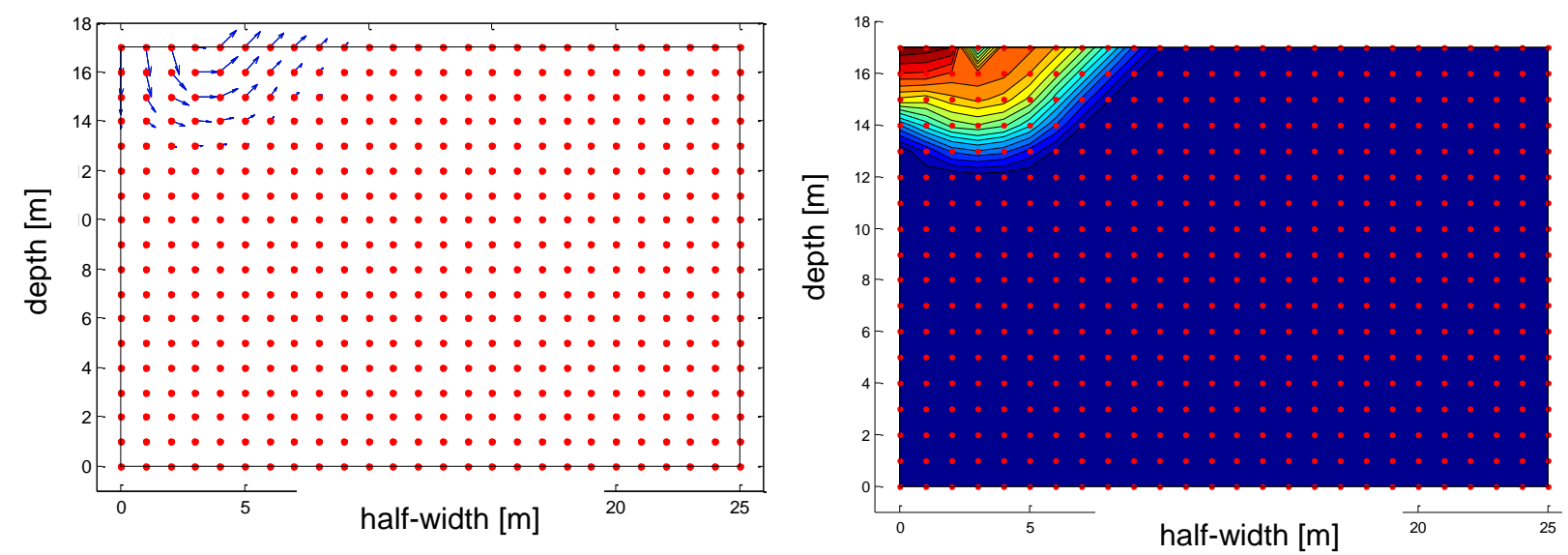

Fig.3: Displacements as vectors and shadings at failure computed by MLFG.

The computed results for this example are given and show the applied stress, the vertical displacement under loaded nodes and the number of iterations at each stage of calculation. These results have been plotted in (Fig. 4 ) in the form of a dimensionless bearing capacity factor $q / c_{u}$ versus centerline displacement. The number of iterations to achieve convergence for each load increment is also shown. It is seen that convergence was achieved in 139 iterations when $q / c_{u}=5.1$, but convergence could not be achieved within the iteration limit of 250 when $q / c_{u}=5.2$. In addition, the displacements are also increasing rapidly at this level of loading, indicating that bearing failure is taking place at a value very close to the Prandtl load of 5.14.

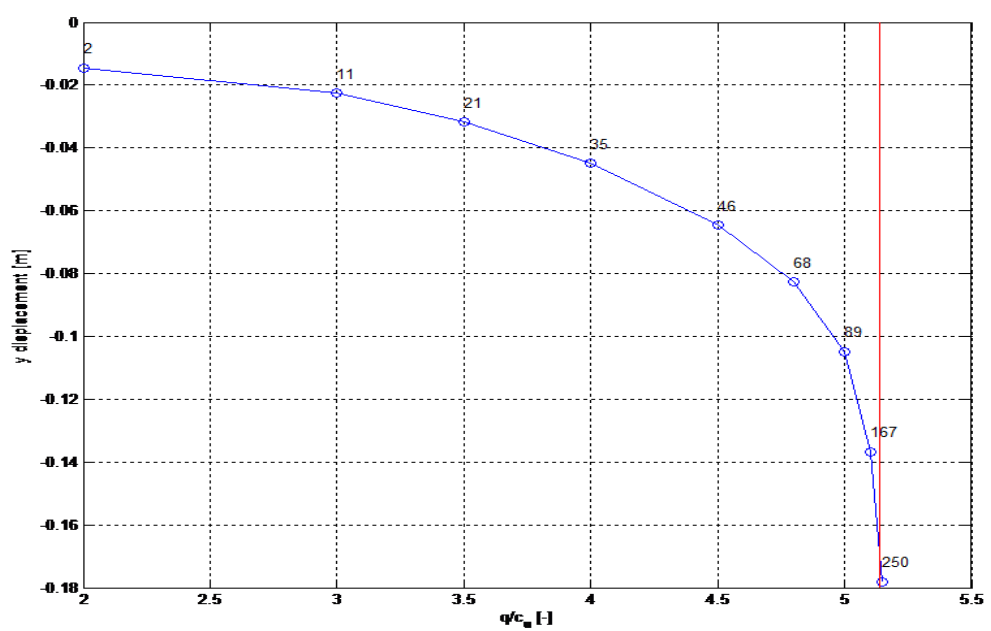

Fig.4: Plot of bearing stress q/cu versuscenterline displacement (Prandtl solution - red line) - MLPG solution.

\section{Conclusions}

The described meshless numerical method MLPG represents alternative to the conventional numerical methods most usually used in geotechnical engineering, like FEM. The area of the meshless methods based on local weak form is still under development. The influence of the nodal meshless shape function types (RPIM, MLS), and shape function parameters should be also investigated with the corresponding types of engineering problems. The article also represents the ability of MLPG to solve the bearing capacity tasks with accuracy comparable with geotechnical FEM implementations. This can be a positive outlook for the application of meshless methods in slope stability analysis. However, as the present study is one of the first 
in this category, it is too soon to conclude generally, and more theoretical and experimental research is needed.

\section{Acknowledgement}

This contribution is the result of the project supported by Scientific Grant Agency of Slovak Republic (VEGA) No. 1-0789-12.

\section{References}

[1] KOVARIK, K.: A meshless solution of two dimensional density-driven groundwater flow. Boundary elements and other mesh reduction methods XXXIII. - Southampton, pp. 253-264, WIT Press, 2011.

[2] GU, Y. T. - LIU, G. R.: A meshless Local Petrov-Galerkin (MLPG) formulation for static and free vibration analyses of thin plates. Computer Modeling in Engineering \& Sciences., pp. 463-476, 2001.

[3] KOVARIK, K.: Numerical simulation of groundwater flow and pollution transport using the dual reciprocity and RBF method. Komunikacie - Communications, pp. 5-10, 3a/2010.

[4] MASAROVIČOVÁ, S.: Testing of rockfill material for soil structures design. In: Theoretical foundation of civil engineering: XXI Russian - Slovak - Polish Seminar: Moscow - Arkhangelsk, 2012. - Warszawa: Politechnika Warszawska, 2012. - ISBN 978-83-7814-021-4., pp. 441-446.

[5] IŽVOLTOVÁ, J. - VILLIM, A.: Aplikácia Gauss-Jacobiho algoritmu pri identifikácii chýb merania. In: Civil and environmental engineering scientific technical journal. - ISSN 1336-5835. - Vol. 8, No. 1 2012, Žilina, pp. 13-17.

[6] DRUSA, M.: Oporné konštrukcie dopravných stavieb, In: Stavební konstrukce z pohledu geotechniky 2008, Brno, Akademické nakladatelství CERM, 2008. - ISBN 978-80-7204-609-6. pp. 41-44.

[7] NGUYEN, G.: An influence of different values of soils shear strength parameters on the size of spread foundation with an inclined eccentric load, In: Theoretical foundation of civil engineering: XXI Russian - Slovak - Polish 2012, Warszawa: Politechnika Warszawska, 2012. ISBN 978-83-7814-021-4. - pp. 451-456. 